\title{
Peripapillary Choroidal Neovascular Membrane Secondary to Sarcoidosis- Related Panuveitis: Treatment with Aflibercept and Ranibizumab with a 50-month Follow-Up
}

\author{
Artemis Matsou $^{a} \quad$ Maria Dermenoudi $^{b}$ Despina Tzetzi ${ }^{b}$ Tryfon Rotsos ${ }^{b}$ \\ Olga Makric Eleftherios Anastasopoulos ${ }^{b}$ Chrysanthos Symeonidis ${ }^{b}$ \\ aDepartment of Ophthalmology, Cambridge University Hospitals, Cambridge, UK; ${ }^{\text {b2 }}$ nd \\ Department of Ophthalmology, Aristotle University of Thessaloniki, Papageorgiou General \\ Hospital, Thessaloniki, Greece; ' Department of Ophthalmology, University General Hospital \\ of Patras, University of Patras, Patras, Greece
}

Keywords

Sarcoidosis - Panuveitis - Peripapillary choroidal neovascular membrane · Anti-vascular endothelial growth factor $\cdot$ Aflibercept $\cdot$ Ranibizumab

\begin{abstract}
A case of peripapillary choroidal neovascular membrane (PCNM) secondary to sarcoidosisrelated panuveitis successfully treated with anti-vascular endothelial growth factor (antiVEGF) agents and systemic immunomodulatory therapy is reported. Diagnosis and follow-up were based on fundoscopic, optical coherence tomography as well as fluorescein angiography findings. A 45-year-old female patient presented with sudden onset bilateral blurring of vision. Fundoscopy revealed bilateral granulomatous panuveitis with solitary peripheral granuloma in the right eye and PCNM in the left eye. Diagnostic work-up including conjunctival biopsy confirmed the diagnosis of sarcoidosis. Topical and systemic corticosteroids controlled the inflammation. Within 4 weeks, PCNM showed rapid enlargement (best-corrected visual acuity [BCVA]: 6/60) with foveal involvement. Monthly intravitreal aflibercept injections and systemic methotrexate were administered. After 5 aflibercept injections, anatomical and functional improvement was noted (BCVA: 6/6). Due to aflibercept unavailability, further treatment included ranibizumab injections. During a 50 -month follow-up period, every anti-VEGF injection was followed by total NV regression and 6/6 BCVA. Both aflibercept and ranibizumab appear to be effective in the treatment of PCNM secondary to sarcoidosis.
\end{abstract}




\section{Introduction}

Sarcoidosis is a chronic, multisystemic, granulomatous, inflammatory disorder of unknown etiology, characterized histologically by non-caseating granulomas in the involved organs, most commonly the lungs, skin, lymph nodes, heart, liver, central nervous system, and eyes [1]. Ocular inflammation is a well-recognized manifestation of the disease since the mid1900 s and can be the presenting symptom in approximately $20-30 \%$ of cases. Uveitis, along with dry eye and conjunctival nodules, has been reported as the most frequently encountered types of ocular sarcoidosis. Choroidal neovascularization (CNV) has been described as a rare but potentially vision-threatening complication of posterior inflammation in sarcoid-related uveitis. The majority of inflammatory CNVs are classic and subfoveal. Timely diagnosis and treatment are imperative and largely determine the ultimate visual outcome. Peripapillary lesions occur less frequently but can have detrimental effects if the subretinal fluid or hemorrhage involves the macula [2]. Various treatment modalities have been employed thus far for the management of uveitis-associated peripapillary choroidal neovascular membranes (PCNMs) such as argon laser photocoagulation [2], photodynamic therapy, subretinal surgery, locally injected, oral corticosteroids, and as mono- or combined therapy. Only a small number of case reports exist in the literature regarding the use of anti-vascular endothelial growth factor (anti-VEGF) agents in sarcoid-related PCNM, with varying and usually short-term results $[3,4]$. We report the case of a patient with bilateral sarcoid panuveitis and a unilateral aggressively progressing PCNM that was managed successfully using 2 anti-VEGF agents (aflibercept and ranibizumab) with a follow-up period of 50 months.

\section{Case Report}

A 45-year-old Greek female patient presented in the 2nd Department of Ophthalmology, Aristotle University of Thessaloniki, Papageorgiou General Hospital, Thessaloniki, Greece, with a 3-day history of bilateral blurry vision and mild ocular discomfort. Past medical history included a hysterectomy for polycystic ovary syndrome and a recent presumed diagnosis of intracranial hypertension, set on the grounds of recurrent headaches and borderline raised intracranial pressure on lumbar puncture. No recent travel abroad or relevant family history was reported. Upon presentation, best-corrected visual acuity (BCVA) was 6/6 bilaterally. Anterior segment examination revealed anterior chamber granulomatous inflammation of 2+ cells with faint flare (SUN working group grading system) [5] bilaterally, mutton-fat keratic precipitates, visible iris Koeppe nodules, and posterior synechiae in 2 clock-h RE. Intraocular pressure was $14 \mathrm{~mm} \mathrm{Hg}$ bilaterally. Dilated fundus examination of RE revealed moderate vitritis of 2+ vitreous haze, a RE peripheral solitary granuloma at 6 o'clock, an optic nerve head granuloma, and choroidal neovascular membrane (PCNM) in the LE with hemorrhage localized in the papillomacular bundle, extending into the macula. Fluorescein angiography and optical coherence tomography (OCT) confirmed biomicroscopy findings; RE: hot disc, LE early staining peripapillary and in the papillomacular bundle (Fig. 1a-d). Laboratory testing (full blood count, clotting, liver screen, viral screen, autoimmune screen, and QuantiferonTB gold) revealed no significant findings with the exception of angiotensin converting enzyme (ACE:148). Thoracic high-resolution computerized tomography revealed irregular nodular thickening predominantly in the middle and upper lung zones bilaterally with no lymphadenopathy, while MRI scans revealed no findings. Blind conjunctival biopsy confirmed sarcoidosis as systemic diagnosis. The patient was followed up by dilated fundoscopy, fluorescein angiography, OCT, and OCT angiography. Unfortunately, indocyanine green angiography was not available in our department during the follow-up period for this patient.

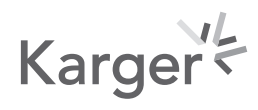



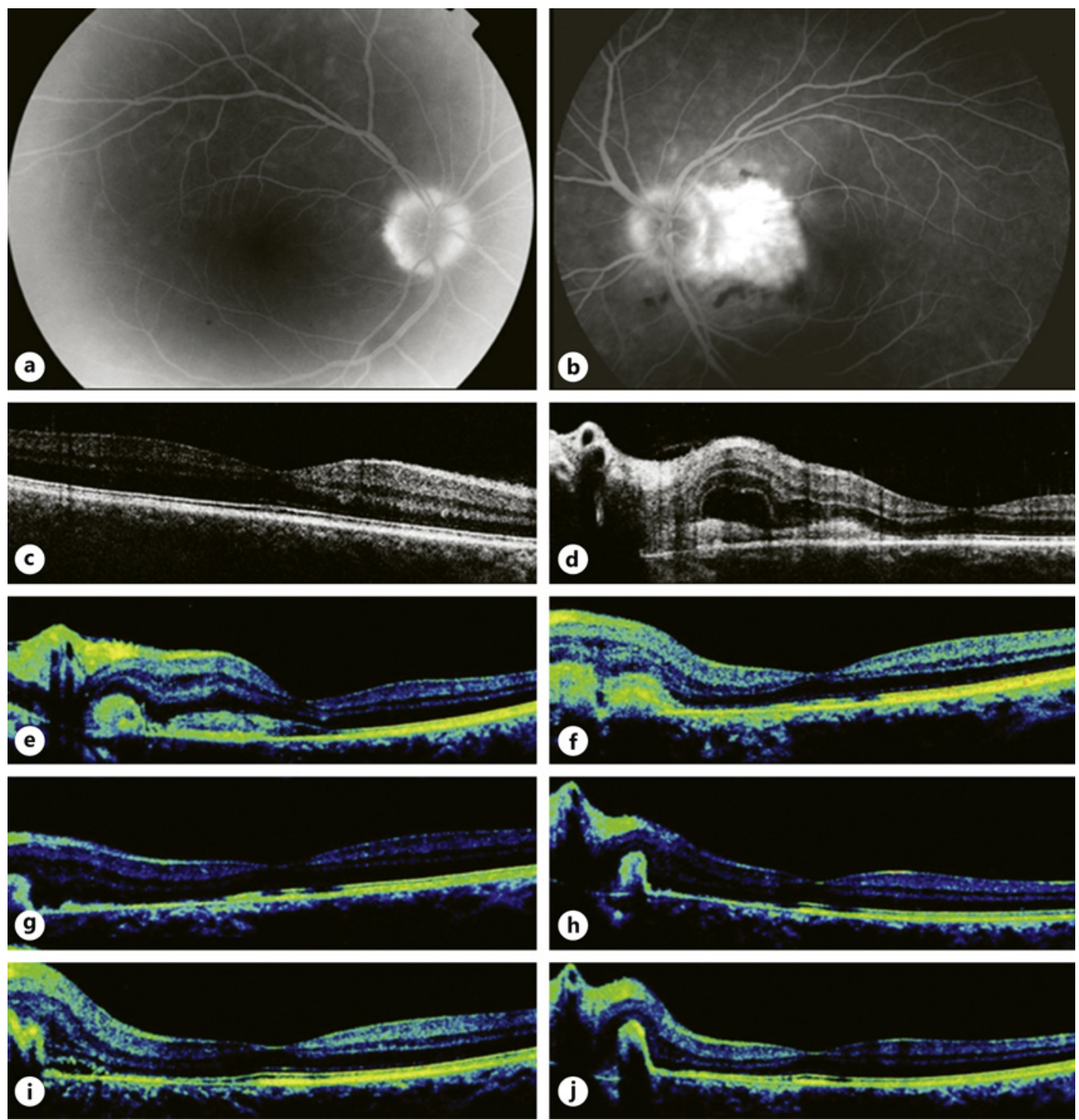

Fig. 1. FA (a, b) and OCT (c, d) depicting (b, d) an active choroidal neovascular membrane with subretinal fluid secondary to sarcoidosis in the left eye at baseline. OCT 4 weeks after the 1st aflibercept injection (e), 2nd (f), 3rd (g), 4th (h), 5th injection (i), and 3 months following the 5th aflibercept injection showing neovascular membrane regression and subretinal fluid absence. FA, fluorescein angiography; OCT, optical coherence tomography.

Within 4 weeks, PCNM showed rapid enlargement (BCVA: 6/60) with foveal involvement. The patient was managed with oral steroids (prednisolone $0.5 \mathrm{mg} / \mathrm{kg}$ ), topical steroid eye drops (dexamethasone $0.1 \%$ ), and cycloplegia in addition to 3 aflibercept injections that were administered in monthly intervals resulting in significant neovascular membrane regression and VA improvement (Fig. 1e-g).

Two more aflibercept injections in monthly intervals followed, due to rapid NV recurrence (Fig. 1h-j). Following the 2nd aflibercept injection, oral steroids were tapered by $5 \mathrm{mg}$ every 2 weeks as per rheumatologist's advice while oral methotrexate was introduced (starting at $7.5 \mathrm{mg} /$ week, increased by $5 \mathrm{mg}$ increments every 2 weeks until a dose of $20 \mathrm{mg} /$

Karger's 
week was reached) with regular monitoring for hepatic and hematologic toxicity. Throughout this 39-month period after oral steroid tapering, there were no significant systemic occurrences that required treatment.

Six months after the onset of treatment and 5 injections, lack of aflibercept availability prompted the use of ranibizumab: 5 injections were administered over 6 months with 5-8-week intervals, each resulting in total NV regression and 6/6 VA. Following a 15-month period of stability, NV recurrence coupled with VA deterioration was observed; over the subsequent 8 months, 4 ranibizumab injections were administered. Finally, the following 16 months were characterized by lack of NV recurrence and 6/6 VA. Overall, during a 50-month follow-up period up to April 2020, on every occasion, an anti-VEGF injection was followed by total NV regression, as depicted by OCT and OCT angiography (Fig. 2) and 6/6 VA.

\section{Discussion}

Sarcoidosis has been reported to be among the most common etiologies of uveitis. Nevertheless, posterior uveitis occurs in a small fraction of sarcoidosis patients: significant visual deterioration occurs in $24 \%$ of the patients with permanent vision loss or handicap in $10 \%$ of these patients [6]. In this context, CNV has been reported as a rare and most commonly subfoveally observed complication. In contrast, peripapillary CNV is less common and more likely to result in severe VA deterioration [2]. Moreover, in the context of this inflammatory neovascular membrane, the deep and outer retinal vascular layers as well as the choriocapillaris were more severely affected during the active phases of the disease compared to the superficial vascular layer, as depicted by OCT angiography. In this case, there were no confounding factors for VA impairment: there was no personal or family ophthalmic history or systemic history with ophthalmic complications. Consequently, PCNV secondary to sarcoidosis and its response to treatment with 2 anti-VEGF agents could be studied without the presence of ophthalmic comorbidities that could alter the overall clinical picture and its course.

The use of intravitreal bevacizumab in the context of PCNV has been shown to result in anatomic improvement in addition to visual acuity retention or even improvement similar to other treatment modalities such as photodynamic surgery, laser photocoagulation, and subretinal surgery [7]. In the context of CNV secondary to uveitis, intravitreal bevacizumab has been found to result in a transient anatomic and functional improvement as reinjection was required in the majority of cases over a period of 18 months (with a mean number of 4.25 injections) [8]. In that report, contrary to our case, the majority of cases (13 out of 15) involved subfoveal $\mathrm{NV}$, an anatomic characteristic that may account for the discrepancy in the mean number of injections required. A comparable mean number of injections (4.45) were required for subfoveal CNV lesions in a similar study over a greater follow-up period of 3 years, while juxtafoveal lesions required significantly fewer injections (2.46) [9].

In the context of PCNV secondary to sarcoidosis, intravitreal ranibizumab has been shown to effectively control NV progression over a period of 12 months with 5 (RE) and 3 injections (LE), respectively [3]. In contrast, in our case, there were 10 injections required over the first 12 months in order to control NV progression. Notable differences between the 2 cases were age of onset ( 44 vs. 81 years), a diagnosis of sarcoidosis of 10 years compared to undiagnosed sarcoidosis and, probably more importantly, NV localization. All these aforementioned parameters may contribute to the increased number of injections required for the control of the PCNV during the first year.

The use of aflibercept has been previously reported in a patient with peripapillary CNV secondary to ocular sarcoidosis [10]. In that case, BCVA decreased significantly following the

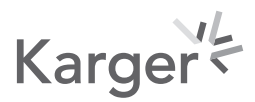



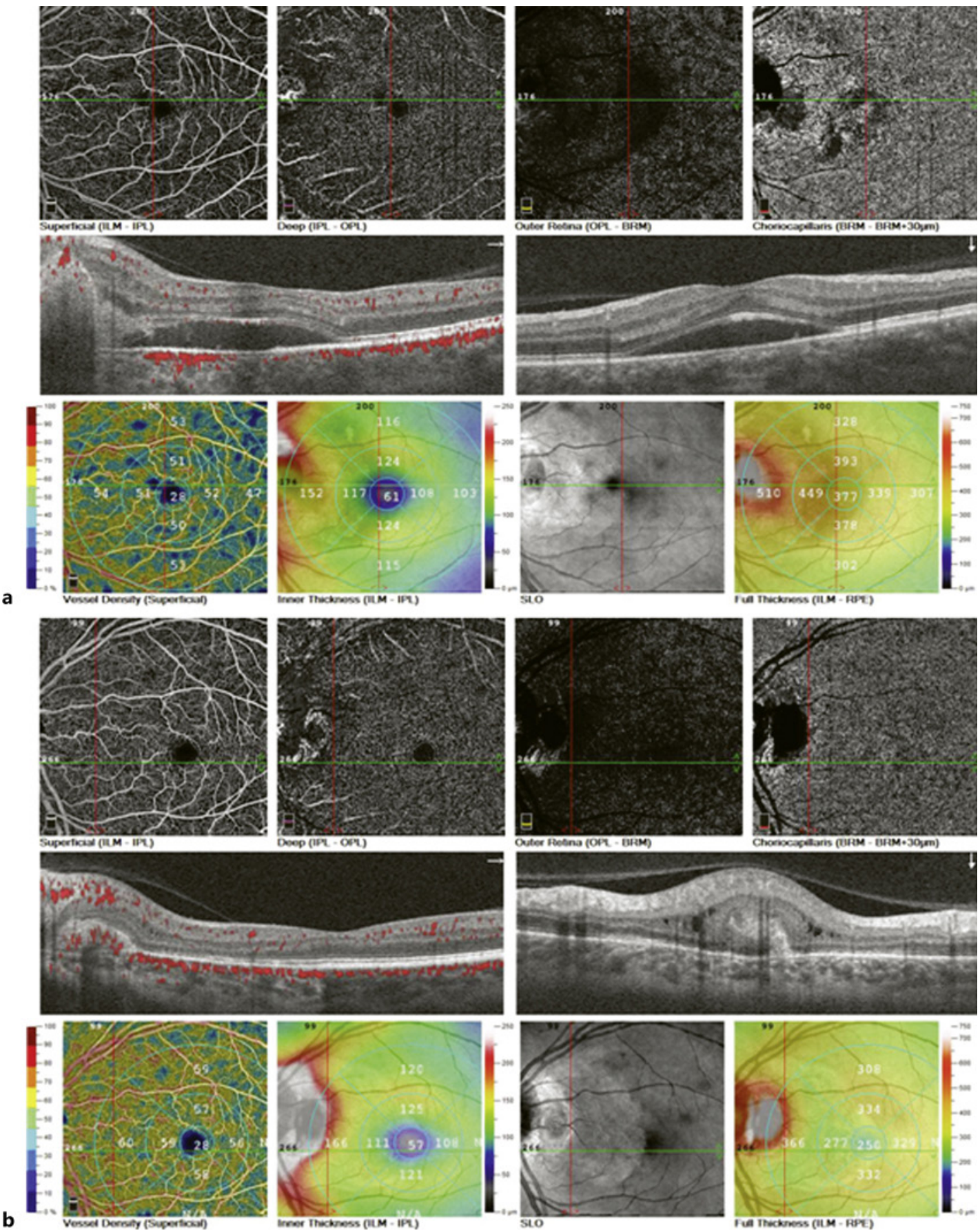

Fig. 2. OCT angiography depicting an active choroidal neovascular membrane with subretinal fluid secondary to sarcoidosis prior to a ranibizumab injection (a) and a preexisting peripapillary granuloma with an intact macula and no activity during follow-up (7 months after the last injection) (b). OCT, optical coherence tomography.

implication of the fovea. In contrast to our case, after a single aflibercept injection, anatomic improvement, and slight functional deterioration was observed, probably due to the initial total loss of the ellipsoid zone. Despite partial recovery of the ellipsoid zone, there was no functional improvement observed 6 months later. In our case, despite temporary macular 
involvement, there was complete resolution of the lesion coupled with full, albeit transient, functional recovery.

In this case, treatment with aflibercept was followed by treatment with ranibizumab due to availability issues. This patient, with no personal or family history of intraocular inflammation and an easily controlled episode of uveitis, appeared to be ideal for observing both anti-VEGF treatments.

During the first year, NV appeared to be more resilient compared to similar cases reported in the relevant literature as 10 anti-VEGF injections were required in order to treat the observed lesion [3]. This increased number of injections could be attributed to the relatively low patient age or to the implication of the fovea as subfoveal inflammatory CNV lesions require more injections compared to juxtafoveal lesions [9]. Conversely, there was no treatment required during the 2 nd and 4 th year, while 4 injections over 8 months were required during the 3rd year.

Aflibercept and ranibizumab use during the first year revealed no significant differences regarding VA, post-injection central retinal thickness, injection intervals, and side effects. Despite their molecular differences, the use of both anti-VEGF agents resulted in NV resolution and VA restoration to its previous pre-inflammation condition (6/6) 2-3 weeks after each injection.

According to our observations, anti-VEGF treatment appears to be effective in the treatment of CNV secondary to sarcoidosis. Stringent follow-up is required especially during the first year as CNV may be more aggressive and, consequently, delayed treatment may result in permanent BCVA decrease.

\section{Statement of Ethics}

Written informed consent was obtained from the patient for publication of this case report and any accompanying images.

\section{Conflict of Interest Statement}

The authors report no proprietary interest or financial support.

\section{Funding Sources}

No funding was attained for the completion of this study.

\section{Author Contributions}

All the authors attest that they meet the current ICMJE criteria for authorship.

\section{Karger'}


Matsou et al.: Sarcoid-Related CNV Treated with Anti-VEGF Agents

\section{References}

1 American Thoracic Society. European Respiratory Society. World Association for Sarcoidosis and Other Granulomatous Disorders. ATS/ERS/WASOG statement on sarcoidosis. Sarcoidosis Statement Committee. Eur Respir J. 1999;14:735-7.

2 Gragoudas ES, Regan CD. Peripapillary subretinal neovascularization in presumed sarcoidosis. Arch Ophthalmol. 1981;99(7):1194-7.

3 Konidaris VE, Empeslidis T. Ranibizumab in choroidal neovascularisation associated with ocular sarcoidosis. BMJ Case Rep. 2013 Nov 14;2013:2013.

4 Shah SP, Hubschman JP, Bourges JL, Hu AY, Schwartz SD. Limited long-term efficacy of intravitreous anti-VEGF pharmacotherapy in sarcoidosis complicated by peripapillary choroidal neovascular membrane. Acta Ophthalmol. 2010;88(6):e243-4.

5 Jabs DA, Nussenblatt RB, Rosenbaum JT, Standardization of Uveitis Nomenclature (SUN) Working Group. Standardization of uveitis nomenclature for reporting clinical data. Results of the First International. Am J Ophthalmol. 2005;140:509-16.

6 Bodaghi B, Touitou V, Fardeau C, Chapelon C, LeHoang P. Ocular sarcoidosis. Presse Med. 2012;41(6 Pt 2): e349-54.

7 Davis AS, Folk JC, Russell SR, Sohn EH, Sohn EH, Boldt HC, et al. Intravitreal bevacizumab for peripapillary choroidal neovascular membranes. Arch Ophthalmol. 2012;130(8):1073-5.

8 Julián K, Terrada C, Fardeau C, Cassoux N, Français C, LeHoang P, et al. Intravitreal bevacizumab as first local treatment for uveitis-related choroidal neovascularization: long-term results. Acta Ophthalmol. 2011;89(2): 179-84.

9 Mansour AM, Arevalo JF, Fardeau C, Hrisomalos EN, Chan WM, Lai TY, et al. Three-year visual and anatomic results of administrating intravitreal bevacizumab in inflammatory ocular neovascularization. Can J Ophthalmol. 2012;47(3):269-74.

10 Querques L, Querques G, Miserocchi E, Modorati G, Bandello F. Intravitreal aflibercept for choroidal neovascularization in ocular sarcoidosis. Eur J Ophthalmol. 2016;26(5):e124-7. 